\title{
Nachhaltige Entwicklungsziele - Der Beitrag der Montanuniversität zur Erstellung eines Optionenpapiers für die österreichische Bundesregierung
}

\author{
Patrick Trummer \\ Resources Innovation Center Leoben, Montanuniversität Leoben, Leoben, Österreich \\ Eingegangen 5. August 2021; angenommen 24. August 2021; online publiziert 29. September 2021
}

\begin{abstract}
Zusammenfassung: Der Beschluss der Vereinten Nationen im Jahr 2015 zu Agenda 2030 für Nachhaltige Entwicklung hat zur Gründung des Projekts UniNEtZ in Österreich geführt. Ziel ist die Erarbeitung von Optionen zur Erreichung der Sustainable Development Goals (SDGs). Somit haben sich österreichische Universitäten und Forschungseinrichtungen aus den verschiedensten Disziplinen zusammengetan, um mit ihrem gemeinsamen Wissen und Erfahrungen Optionen gemeinsam zu entwickeln. Die Montanuniversität Leoben mit der Expertise im Bereich Rohstoffe, Werkstoffe und deren Verarbeitung ist dadurch ein wichtiger Partner. Das Resources Innovation Center Leoben als Teil der Montanuniversität ist federführenden bei SDG 7 und SDG 12. Die Thematik der Ressourcen speziell der mineralischen Rohstoffe adressiert das Subziel 12.2 und ist ebenso ein wesentlicher Bestandteil einer Kreislaufwirtschaft, da Rohstoffe eine essentielle Rolle in einer industrialisierten Welt spielen. Eine effiziente und effektive Nutzung von Ressourcen stellt eine Schüsselfunktion für eine Nachhaltige Entwicklung dar. Daraus entstand im SDG 12 eine Option zum Thema Rohstoffe, die sich speziell am Beginn der Kreislaufwirtschaft platziert. Ziele sind die nachhaltige Gestaltung von Produktionsprozessen, die ganzheitliche Verwendung von Rohstoffen sowie ein nachhaltiges und intelligentes Rohstoffmanagement zu forcieren.
\end{abstract}

Schlüsselwörter: Agenda 2030, SDG, UniNEtZ, Kreislaufwirtschaft, Mineralische Rohstoffe, Optionen, Nachhaltige Entwicklung, Nachhaltige Produktion und Konsum

$\overline{\text { Dipl.-Ing. P. Trummer ( }} \varangle$ )

Resources Innovation Center Leoben,

Montanuniversität Leoben,

Franz-Josef-Straße 18,

8700 Leoben, Österreich

patrick.trummer@unileoben.ac.at
Sustainable Development Goals-The Contribution of the University of Leoben to the Preparation of an Options Paper for the Austrian Federal Government

Abstract: The decision of the United Nations in 2015 on Agenda 2030 for Sustainable Development has led to the establishment of the project UniNEtZ in Austria. The goal is to develop options for achieving the Sustainable Development Goals (SDGs). Thus, Austrian universities and research institutions from a wide range of disciplines have joined forces to use their collective knowledge and experience to jointly develop options. The University of Leoben with its expertise in the field of raw materials, materials, and their processing is an important partner. The Resources Innovation Center Leoben, as part of the University of Leoben, is in charge of SDG 7 and SDG 12. The topic of resources, especially mineral raw materials, addresses target 12.2 and is also an essential component of a circular economy as raw materials play an essential role in an industrialized world. An efficient and effective use of resources is a key function for a sustainable development. This resulted in an option on raw materials in SDG 12 , which is specifically placed at the beginning of the circular economy. The goals are to promote the sustainable design of production processes, the holistic use of raw materials, and a sustainable and intelligent raw materials management.

Keywords: Agenda 2030, SDG, UniNEtZ, Circular economy, Mineral resources, Options, Sustainable development, Sustainable production and consumption

\section{Nachhaltige Entwicklung - Projekt UniNEtZ+SDGs}

Die Organisation der Vereinten Nationen (UNO) hat im September 2015 die Agenda 2030 für Nachhaltige Entwicklung beschlossen. Somit sind alle Mitgliedstaaten der UNO in 
der Pflicht, auf die Umsetzung der 17 Sustainable Development Goals (SDGs) (Ziele für Nachhaltige Entwicklung) bis 2030 hinzuarbeiten. Hierfür hat der Staat Österreich per Ministerratsbeschluss von 12. Jänner 2016 sämtliche Bundesministerien zur Implementierung der Agenda 2030 beauftragt.

Eine wichtige Rolle bei der Umsetzung der SDGs spielen die Universitäten. Dieser Impuls sorgte für die Entwicklung eines gemeinsamen Projektes der Allianz Nachhaltiger Universitäten in Österreich unter dem Namen UniNEtZ- Universitäten und Nachhaltige Entwicklungsziele.

An diesem Projekt sind aktuell 18 Universitäten und Forschungseinrichtungen beteiligt - mit dem Ziel der Erstellung eines Optionenpapiers für die österreichische Bundesregierung. Dieses Optionenpapier erfasst an der Zukunft orientierte wissenschaftliche Beiträge als Handlungsoptionen im Sinne der umzusetzenden Agenda 2030 und arbeitet diese aus. Des Weiteren soll nachhaltige Entwicklung an den Universitäten in Lehre und Forschung verankert werden.

Die Montanuniversität Leoben (MUL) beteiligt sich zusammen mit anderen Universitäten am UniNEtZ an den SDGs 4 (Hochwertige Bildung), 7 (Leistbare und saubere Energie), 12 (Nachhaltige Produktion und Konsum) und SDG 13 (Maßnahmen zum Klimaschutz). Das Resources Innovation Center Leoben (RIC) befasst sich im Rahmen dieser Initiative schwerpunktmäßig mit den folgenden Entwicklungszielen:

- SDG 12: Nachhaltige Produktion und Konsum

- SDG 13: Maßnahmen zum Klimaschutz

\section{Allgemeines zu SDG 12 aus UniNEtZ}

Das SDG 12 „Nachhaltige Konsum- und Produktionsmuster sicherstellen" [1] zielt darauf ab, nachhaltige Konsumund Produktionsmuster zu entwickeln sowie deren Wettbewerbsfähigkeit, Realisierbarkeit und Praktikabilität sicherzustellen. Um die Unterziele 12.1-12.8 und 12.a-12.c zu erreichen, ist die Anwendung eines breiten Instrumentariums notwendig, welches sowohl die Unternehmen der produzierenden Industrie, Service- und Handelsunternehmen in der gesamten Wertschöpfungskette beinhaltet als auch die Endkonsument_innen miteinbezieht. Expert_innen sind sich dabei einig, dass weitreichende - und nicht bloß inkrementelle oder konventionelle - gesellschaftliche und wirtschaftliche Veränderungen notwendig sein werden, die direkt die Probleme einer weltweit steigenden Konsumption, der Emission von Treibhausgasen und der wachsenden Ungleichheit und Armut aufgreifen [2, 3].

Die Targets, die im Rahmen von SDG 12 berücksichtigt werden, sind:

- 12.2: Die effiziente und effektive ${ }^{1}$ Nutzung der natürlichen Ressourcen

\footnotetext{
1 Anmerkung: kursiv geschriebene Textpassagen sind als Ergänzung zu den von der UN definierten Zielen zu verstehen, die von der SDG 12 Arbeitsgruppedefiniertwurden.
}

- 12.3: Die Reduktion der Nahrungsmittelverschwendung sowie die bestmögliche Nutzung nicht vermeidbarer Lebensmittelabfälle

- 12.4: Ein umweltverträglicher Umgang mit Abfällen und Chemikalien

- 12.5: Die Reduktion des Abfallaufkommens durch Vermeidung, Verminderung, Wiederverwendung und Wiederverwertung

- 12.6: Die Einführung nachhaltiger betrieblicher Aktivitäten und Maßnahmen sowie Berichterstattung darüber durch Unternehmen

- 12.7: Nachhaltige Beschaffung

- 12.8: Die Förderung des Bewusstseins und vor allem auch des Handelns im Sinne eines nachhaltigen Lebensstils in der Bevölkerung

Der Fokus der Bearbeitung von SDG 12 wird auf die oben beschriebenen Targets 12.2-12.8 gesetzt. Als Bezugsrahmen und konzeptionelle Brücke für diese Targets wird dabei die Kreislaufwirtschaft herangezogen [3].

Die methodische Erarbeitung der Handlungsoptionen für SDG 12 erfolgte in der Arbeitsgruppe durch Wissensund Informationsaustausch in sieben SDG 12-Workshops und zahlreichen Target-bezogenen Workshops sowie durch Forecasting- und Backcasting-Methoden. Erstere basieren auf der Analyse relevanter bestehender Initiativen und Programme (\#mission 2030, Ref-NEKP, österreichisches Regierungsprogramm 2020, European Green Deal, New Circular Economy Action Plan) und der Strukturierung, Bewertung sowie Ergänzung dieser. Aus der Kombination dieser Szenario-Techniken ergab sich ein Pool an möglichen Optionen, aus dem das finale Optionen-Set ausgewählt wurde (siehe Abb. 1). Die Ausarbeitung der Optionen erfolgte in Sub-Arbeitsgruppen [3].

Um für dieses breite Themenfeld und die relevanten Akteur_innen (Produzenten, Handel, Konsument_innen, Unternehmen der Abfall- und Recyclingwirtschaft) eine gemeinsame Zielorientierung zu schaffen und eine kongruente Zielintegration zu ermöglichen, ist ein gemeinsamer Bezugsrahmen notwendig (siehe Abb. 2). Sowohl aufgrund der wesentlichen regulatorischen Entwicklungen als auch aufgrund der inhaltlichen Abdeckung bietet sich hierzu das Konzept der Zirkulärwirtschaft bzw. Kreislaufwirtschaft an. Das übergeordnete Ziel der Kreislaufwirtschaft (engl. Circular Economy) ist es, mit den natürlichen Ressourcen effizienter umzugehen sowie auch eine bessere Kompatibilität der eingesetzten und genutzten Stoffe mit der natürlichen Umwelt zu erreichen. In der EU werden seit Ende 2014 durch die Beschließung des 1. Circular Economy Packages (,Towards a circular economy: a zero waste programme for Europe“) und mit dem im März 2020 neu veröffentlichten „New Circular Economy Package“ wesentliche Weichen für Nachhaltige(n) Konsum und Produktion gestellt $[4,5]$. Hierbei wird ein breiter Ansatz vertreten, der weit über das stoffliche Recycling (so genannte "Closing"-Strategien) hinausgeht und auch die Produktlebensdauer und damit verbundene Konsumgewohnheiten (so genannte "Slowing"-Strategien) adressiert [6, 7]. Im folgenden Circular Economy (CE) Framework als Bezugsrahmen werden die Abdeckung, die Systemgrenzen und der Zusammenhang der einzelnen 


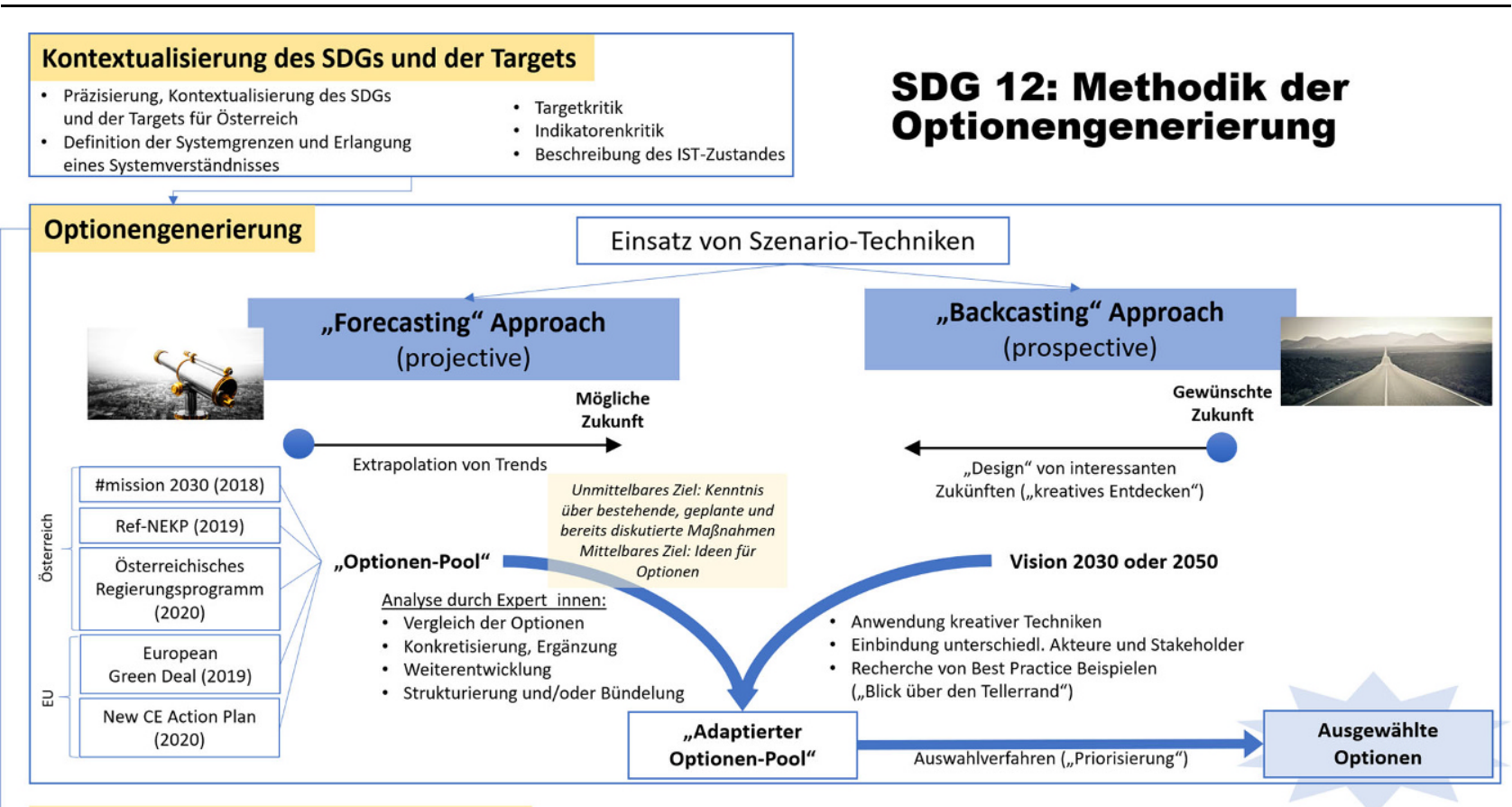

\section{Optionen- und Interaktionenbewertung}

Abb. 1: Methodische Vorgehensweise im Rahmen von SDG 12. Quelle: Eigene Darstellung [3]

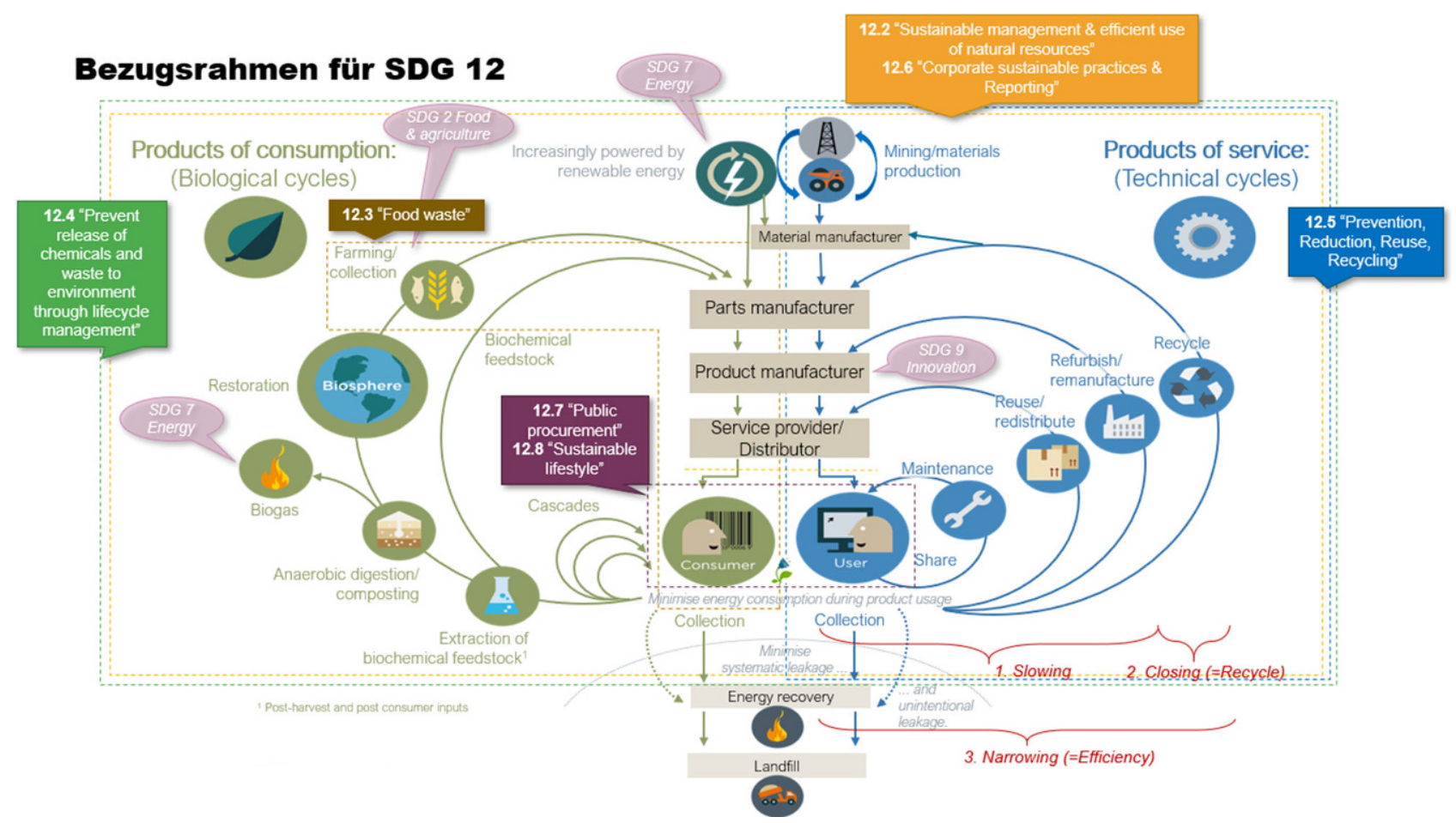

Abb. 2: Bezugsrahmen für SDG-12 anhand des Circular Economy Frameworks der Ellen MacArthur Foundation [8]

Targets sichtbar (dargestellt als strichlierte färbige Linien mit den entsprechenden Verweisen auf die jeweiligen Targets) [3].

Die Grundidee der CE basiert auf dem aus den 1970erJahren bekannten Konzept der Kreislaufwirtschaft, welches heute als produktlebenswegbezogene Innovationsstrate- gie neu interpretiert wird [9-12]. Der CE-Ansatz steht im Gegensatz zur traditionellen linearen Stoffdurchflusswirtschaft (take-make-use-dispose) und berücksichtigt technische Kreisläufe für Gebrauchsprodukte und biologische Kreisläufe für Verbrauchsprodukte $[6,13]$ im Kontext zunehmender Nutzung erneuerbarer Energien [3]. 
Abb. 3: Derösterreichische Materialverbrauch (DMC) nach Materialkategorien, 2018 [16]

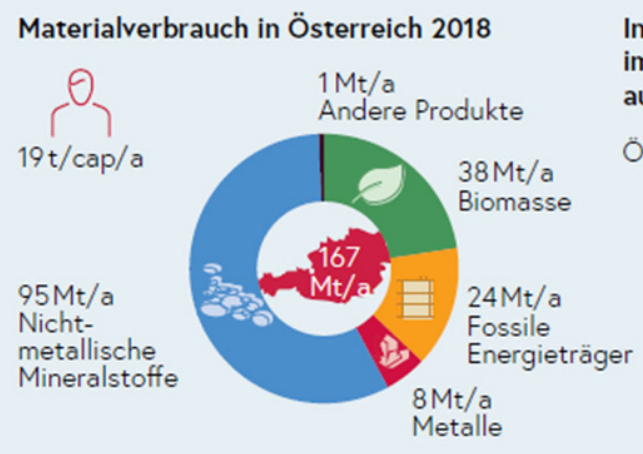

In Bezug auf den DMC lag Österreich im europäischen Vergleich im Jahr 2018 auf dem 11. Platz

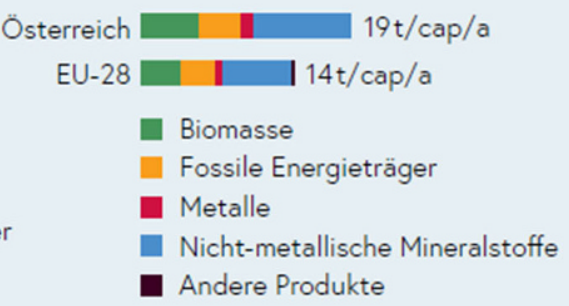

Werte sind gerundet, Rundungsdifferenzen wurden nicht ausgeglichen

Materialverbrauch (DMC, "domestic material consumption")= Inlandsentnahme+Importe-Exporte
Der beschriebene Bezugsrahmen, der zugleich die inhaltliche Systemgrenze für das SDG 12 darstellt, umfasst deshalb sowohl eine Produkt- und Nutzungs- als auch eine Standortperspektive und bezieht sämtliche daran beteiligten und betroffenen Akteur_innen mit ein. Damit einhergehend verstehen wir den Bezugsrahmen der CE als einen, der alle drei Dimensionen der Nachhaltigkeit in einer integrierten Sichtweise miteinbezieht (embedded view) und damit die häufig isolierte Sichtweise der so genannte "drei Säulen“ der Nachhaltigkeit aufzuheben versucht [14]. Der Bezugsrahmen fokussiert damit auf ökologische und soziale Sachverhalte, etwa im Bereich der Zulieferkette von Produkten (d.h. unter welchen ökologischen und sozialen Rahmenbedingungen werden Materialien abgebaut, verarbeitet und Produkte hergestellt), sowie in der Nutzungsphase, wo ebenfalls soziale und auch psychologische Aspekte eine wesentliche Rolle spielen. Im Rahmen der CE wird die Ökonomie (im Sinne des wirtschaftlichen Handelns) einerseits als Instrument verstanden, diese ökologischen und sozialen Sachverhalte entsprechend umzusetzen, andererseits wirkt sich eine sinnvoll umgesetzte $C E$ auch positiv auf ökonomische Aspekte. Alle Akteure (Unternehmen, Konsument_innen, öffentliche Hand, NGOs) können durch entsprechend aufeinander abgestimmte Handlungsweisen zu Veränderungen im Sinn der SDGs beitragen. Hierzu braucht es Rahmenbedingungen, die innovatives Handeln, Pioniergeist, Risikobereitschaft und neue Kooperationsformen aller diesen Akteursgruppen fördern [3, 15].

Die räumliche Systemgrenze ist im österreichischen Kontext und Verantwortungsbereich angesiedelt. In den heutzutage üblichen globalen Wertschöpfungsketten können nicht immer alle Faktoren in den Zulieferketten beeinflusst werden. Die Grenze für SDG 12 ist also hier zu ziehen, wo österreichische Unternehmen und Akteure keinen Wirkungseinfluss mehr auf vor- oder nachgelagerte Lieferketten haben. Die zeitliche Systemgrenze reicht über das Jahr 2030 hinaus, da die Erreichung einiger der Targets von SDG 12 langfristige Maßnahmen erfordern [3].

\section{Target 12.2}

Die Montanuniversität Leoben mit ihrer langjährigen Expertise im Bereich Rohstoffe und Werkstoffe befasst sich speziell mit dem Subziel 12.2 (Die effiziente und effektive Nutzung der natürlichen Ressourcen). Da die Thematik Ressourcen ein sehr breites Feld aufweist, wurde der Fokus speziell auf mineralische Rohstoffe gelegt, da dies auch den größten Teil der Ressourcennutzung in Österreich ausmacht. (siehe Abb. 3). Im Jahr 2018 wurden ca. 103 Mt/a an mineralischen Rohstoffen (Nichtmetallische und metallische Rohstoffe) in Österreich verbraucht und untermauert den zuvor erwähnten Fokus [3].

Da jedoch Rohstoffe in einer Kreislaufwirtschaft einen langen Weg mit vielen Akteuren_innen und verschiedenen Bereichen aufweist, war eine zusätzliche Fokussierung nötig, da die weiteren Subziele des SDG 12 sich ebenfalls mit der Thematik Rohstoffe befassen, wie bspw. Recycling, Konsum, Beschaffung und mehr. Diese betrachten jedoch den Rohstoff nur innerhalb der Kreislaufwirtschaft. Es ist wichtig zu verstehen, dass Rohstoffe laufend in eine Kreislaufwirtschaft eingeführt werden müssen, damit Waren, Güter und Produkte hergestellt und wiederverwertet werden können. Daher liegt das Hauptaugenmerk des Subziels 12.2 auf den Bereich vor der Kreislaufwirtschaft, von der Exploration über die Gewinnung bis zur Herstellung von Halbfabrikaten, wie bspw. Rohre, Schienen, Bleche und mehr. Abb. 4 zeigt die Systemgrenze innerhalb des Subziels 12.2. Die Einteilung erfolgt in Primär- und Sekundärsektoren. Der Primärsektor bezieht sich auf den klassischen Bergbau. Im Anschluss werden die gewonnen Rohstoffe in den jeweiligen Industrien zu Werkstoffen, Baumaterialien, Verpackungen und mehr weiterverarbeitet. Der Sekundärsektor bezieht sich auf die Gewinnung von Rohstoffen aus dem Recycling und der Wiederaufbereitung. Zusätzlich wurden noch mögliche Potenziale der Rohstoffgewinnung ermittelt [3].

Mit den damit definierten Rahmen ( $C E$, mineralische Rohstoffe, Systemgrenze) wurden Maßnahmen zur Erreichung des Subziels 12.2 entwickelt. Die einzelnen Maßnahmen sind zu drei Maßnahmenkombinationen gebündelt, 
Abb. 4: Systemgrenze von Target 12.2. Quelle: Eigene Darstellung [3]

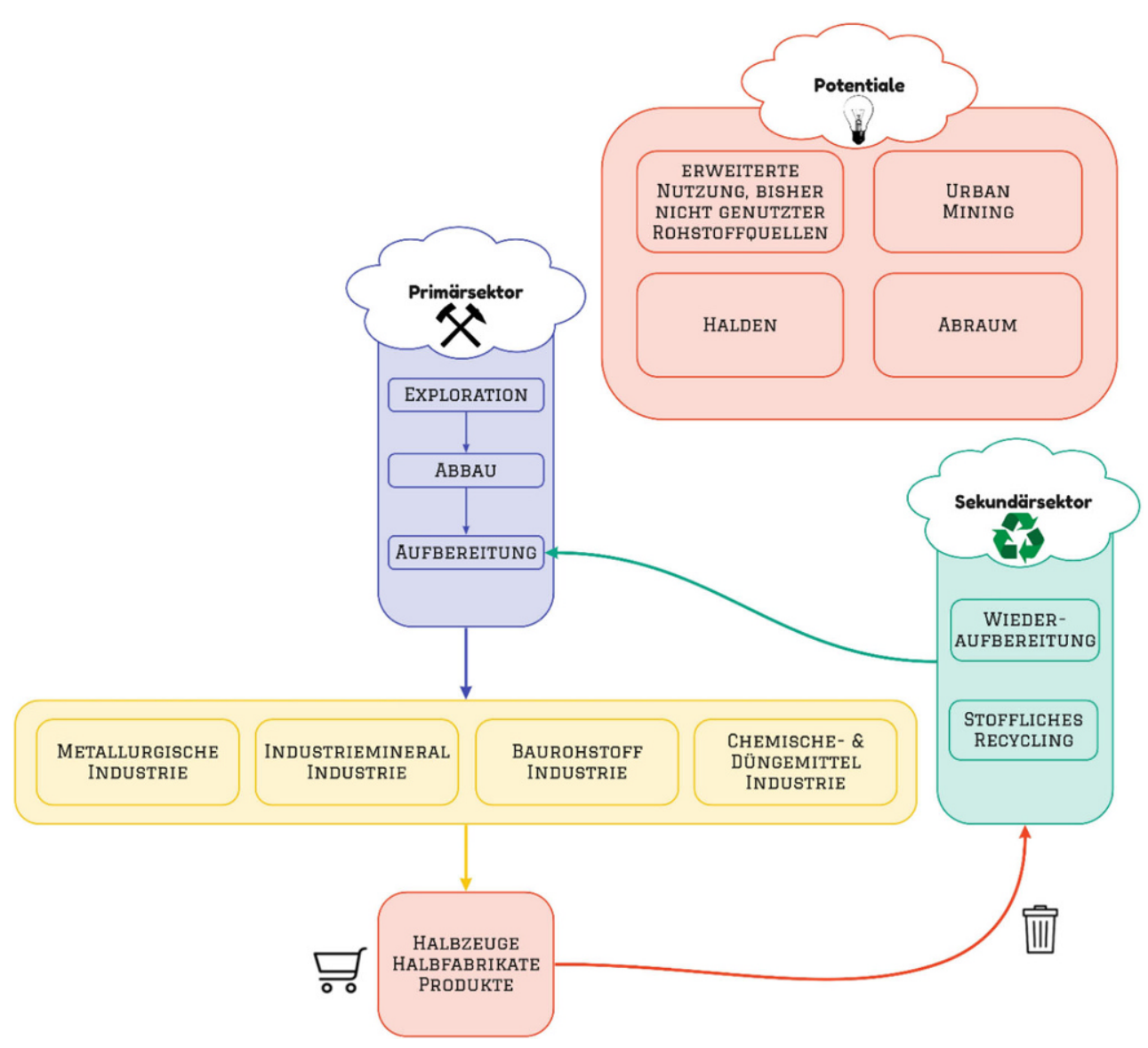

die eine nachhaltige Entwicklung der einzelnen Schritte des Rohstoffweges (von der Exploration über den Abbau bis hin zur Herstellung von Halbfabrikaten) fördern, wobei der Fokus auf folgenden Bereichen liegt.

- Nachhaltige Gestaltung von Produktionsprozessen der rohstoffverarbeitenden Industrie

- Ganzheitliche Verwendung von Rohstoffen und alternativen Rohstoffquellen

- Nachhaltiges und intelligentes Rohstoffmanagement

Gesamt wurden im SDG 12 sieben Optionen mit Maßnahmen entwickelt, um die einzelnen Subziele von SDG $12 \mathrm{zu}$ erreichen.

- Option 1: Nachhaltiger Umgang mit mineralischen Rohstoffen von der Gewinnung bis inklusive Halbzeugherstellung

- Option 2: Aktionsplan Hochwertige Recycling: Design for Recycling, Schadstofffreiheit \& Einsatz von Sekundärrohstoffen

- Option 3: Reduzierung von Lebensmittelverlusten

- Option 4: Änderung des Abfallregimes (Beginn und Ende der Abfalleigenschaft) zur Verlängerung der Kreislaufwirtschaft

- Option 5: Forcierung nachhaltiger Unternehmen
- Option 6: Integration von Ökobilanzen in öffentliche Bau-Ausschreibungsverfahren unter Berücksichtigung der Pre- und Post-Procurement-Phase

- Option 7: Konsum von Gebrauchsgütern in einer Kreislaufwirtschaft: nachhaltig und transformativ

Funding. Open access funding provided by Montanuniversität Leoben.

Open Access Dieser Artikel wird unter der Creative Commons Namensnennung 4.0 International Lizenz veröffentlicht, welche die Nutzung, Vervielfältigung, Bearbeitung, Verbreitung und Wiedergabe in jeglichem Medium und Format erlaubt, sofern Sie den/die ursprünglichen Autor(en) und die Quelle ordnungsgemäß nennen, einen Link zur Creative Commons Lizenz beifügen und angeben, ob Änderungen vorgenommen wurden.

Die in diesem Artikel enthaltenen Bilder und sonstiges Drittmaterial unterliegen ebenfalls der genannten Creative Commons Lizenz, sofern sich aus der Abbildungslegende nichts anderes ergibt. Sofern das betreffende Material nicht unter der genannten Creative Commons Lizenz steht und die betreffende Handlung nicht nach gesetzlichen Vorschriften erlaubt ist, ist für die oben aufgeführten Weiterverwendungen des Materials die Einwilligung des jeweiligen Rechteinhabers einzuholen.

Weitere Details zur Lizenz entnehmen Sie bitte der Lizenzinformation auf http://creativecommons.org/licenses/by/4.0/deed.de. 


\section{Literatur}

1. Vereinte Nationen (UN): Transforming our World: The 2030 Agenda for Sustainable Development (A/RES/70/1), 2015, S. 24, http://www. un.org/Depts/german/gv-70/band1/ar70001.pdf (01.04.2021)

2. Randers, J.; Rockström, J.; Stoknes, P. E.; Goluke, U.; Collste, D.; Cornell, S. E. et al: (2019). Achieving the 17 Sustainable Development Goals within 9 planetary boundaries. Global Sustainability, 2 (2019), pp 1-11, https://doi.org/10.31223/osf.io/xwevb

3. UniNEtZ: Österreichs Handlungsoptionen für eine lebenswerte Zukunft. Die UN-Agenda 2030 aus Sicht der Wissenschaft, 2021

4. Europäische Kommission (EC) (Hrsg.): Mitteilung der Kommission an das Europäische Parlament, den Rat, den Europäischen Wirtschafts- und Sozialausschuss und den Ausschuss der Regionen. Hin zu einer Kreislaufwirtschaft: Ein Null-Abfallprogramm für Europa $\operatorname{COM}(2014) 398$ final/2, 2014

5. Europäische Kommission (EC) (Hrsg.): Circular Economy Action Plan, 2020 https://ec.europa.eu/environment/circular-economy/pdf/ new_circular_economy_action_plan.pdf (02.04.2021)

6. Bocken, N. M. P.; de Pauw, I., Bakker, C.; van der Grinten, B.: Product design and business model strategies for a circular economy, Journal of Industrial and Production Engineering, 33 (2016), no 5, pp 308-320, https://doi.org/10.1080/21681015.2016.1172124

7. Hansen, E.G.; Revellio, F: Circular value creation architectures: Make, ally, buy, or laissez-faire, Journal of Industrial Ecology, 24 (2020), no 6, pp 1250-1273, https://doi.org/10.1111/jiec.13016

8. Ellen MacArthur Foundation (EMF). (Hrsg.) (2013). Towards the Circular Economy 1: Economic and business rationale for an accelerated transition. http://ellenmacarthurfoundation.org/ (02.04.2021)

9. Hansen, E.G.; Schmitt, J.: Circular Economy: Potenziale für Produkt- und Geschäftsmodellinnovation heben. UC Journal, 2016, no 2(2-October), pp 8-10, http://www.cleantech-cluster.at/fileadmin/ user_upload/Cluster/UC/Downloadunterlagen/UC_Journal_2_2016_ download_web.pdf. https://doi.org/10.13140/RG.2.2.31518.54081

10. Hansen, E. G.; Schmitt, J. Orchestrating Cradle-to-Cradle Product Innovation Across the Value Chain: Innovation Community Evolution,
Collaboration Mechanisms, and Intermediation. Journal of Industrial Ecology, 2020, pp 1-21, https://doi.org/10.1111/jiec.13081

11. Stahel, W. R.: Ressourcenproduktivität durch Nutzungsintensivierung und Lebensdauerverlängerung - 10 Jahre Good Practice Beispiele, Norderstedt: Books on Demand GmbH, 2004

12. Stahel, W. R.; Giarini, O.: Die Performance-Gesellschaft: Chancen und Risken beim Übergang zur Service Economy, Marburg: Metropolis, 2000

13. Braungart, M.; McDonough, W.; Bollinger, A.: Cradle-to-cradle design: creating healthy emissions - a strategy for eco-effective product and system design, Journal of Cleaner Production, 15 (2007), no (13-14), pp 1337-1348 https://doi.org/10.1016/j.jclepro.2006.08. 003

14. Marcus, J.; Kurucz, E. C.; Colbert, B. A.: Conceptions of the Business-Society-Nature Interface: Implications for Management Scholarship, Business \& Society, 49 (2010), no 3, pp 402-438, https://doi. org/10.1177/0007650310368827

15. acatech - Deutsche Akademie für Technikwissenschaften (2020). Circular Business Models: Overcoming Barriers, Unleashing Potentials: Report of the Working Group on Circular Business Models, Circular Economy Initiative Deutschland. Executive Summary and Recommendations. acatech - Deutsche Akademie der Technikwissenschaften e.V. (acatech); Circular Economy Initiative Deutschland (CEID); SYSTEMIQ Ltd., München. https://en.acatech.de/publication/ circular-business-models-overcoming-barriers-unleashing-poten tials/ (02.04.2021)

16. Bundesministerium für Klimaschutz, Umwelt, Energie, Mobilität, Innovation und Technologie: Ressourcennutzung in Österreich 2020, 2020, https://www.bmk.gv.at/themen/klima_umwelt/nachhaltigkeit/ ressourceneffizienz/publikationen/bericht2020.html (13.03.2021)

Hinweis des Verlags. Der Verlag bleibt in Hinblick auf geografische Zuordnungen und Gebietsbezeichnungen in veröffentlichten Karten und Institutsadressen neutral. 